\title{
An integrated Silicon Drift Detector System for FEI Schottky Field Emission Transmission Electron Microscopes
}

\author{
H.S. von Harrach*, P. Dona*, B. Freitag*, H. Soltau**, A. Niculae**, M. Rohde*** \\ *FEI Company, Achtseweg Noord 5, 5600 KA Eindhoven, The Netherlands \\ **PN Sensor GmbH, Otto-Hahn-Ring 6, D-81739 Munich, Germany \\ ***Bruker AXS Microanalysis GmbH, Schwarzschildstr. 12, 12489 Berlin, Germany
}

Silicon Drift Detectors (SDD) [1] are rapidly replacing $\mathrm{Si}(\mathrm{Li})$ detectors for EDX microanalysis in SEM, but have yet to have an impact in the S/TEM world. Main reason for this difference is the low count rate created by thin S/TEM samples compared to the bulk samples in SEM . These low count rates make EDX mapping a very slow process in $\mathrm{S} / \mathrm{TEM}$. However, the recent introduction of higher brightness electron sources [2] and probe Cs-correctors has led to significantly increased beam currents in small electron probes and, potentially, to higher EDX count rates. Since a key advantage of the SDD is the high count rate capability, the throughput improvement compared to the $\mathrm{Si}(\mathrm{Li})$ detectors will be considerable in these new instruments.

Compared to SEM, the smaller excited volumes obtained with the atomic-scale probes in the new S/TEM instruments can lead to radiation damage of beam-sensitive materials before the analysis is completed. Therefore S/TEM microanalysis needs not only the higher count rate capability, but also higher collection efficiency of the X-rays generated, in order to reduce the dose on the sample.

In this paper we present a new prototype EDX detector system for an FEI $200 \mathrm{kV}$ TEM/STEM, in which FEI has integrated a detector system consisting of multiple SDDs, placed symmetrically around the electron beam axis in the objective lens chamber without affecting the S/TEM resolution. The SDDs with a total active area of $120 \mathrm{~mm}^{2}$ were designed by PN Sensor to fit into the FEI design to achieve a quantum leap in solid angle of collection compared to previous designs in S/TEMs. The SDDs are cooled to achieve the optimum energy resolution, typically below $130 \mathrm{eV}$. The windowless design allows for better sensitivity for light-element detection than conventional thin-window detectors. The specially designed front-end electronics and ultra fast multi-channel pulse processor are provided by Bruker AXS MA in collaboration with FEI. The processor is capable of fast mapping with pixel dwell times down to a few microseconds and $>100$ kcps count rates per channel.

Compared to currently available $\mathrm{Si}(\mathrm{Li})$ detectors the anticipated count rates will be an order of magnitude higher with the new detector. Additionally the new high brightness gun of FEI (X-FEG) [2] increases the brightness of the electron source compared to conventional Schottky sources, leading to a further increase in count rate, and an equivalent significant decrease in mapping time at the same spatial resolution. This improvement is illustrated in Fig. 1 where the relative minimum detectable mass MDM (t.P.P/B $)^{-1 / 2}$ ( $\mathrm{t}=$ analysis time, $\mathrm{P}=$ elemental peak counts, $\mathrm{P} / \mathrm{B}=$ peak-to-background ratio) [3] is shown for conventional and new EDX detector count rates at the same spatial resolution. Fig.1 also compares the MDM with EELS and, for the specific case of strontium titanate, shows that the new EDX detector is expected to be more sensitive than EELS. Further results will be reported at the conference. 


\section{References}

[1] E. Gatti and P. Rehak, Nucl. Instrum. Methods A 225 (1984) 608

[2] B. Freitag et al., Microsc. Microanal. 14 (Suppl.2) (2008) 1370.

[3] T.O. Ziebold, Anal. Chem. 39 (1967) 859

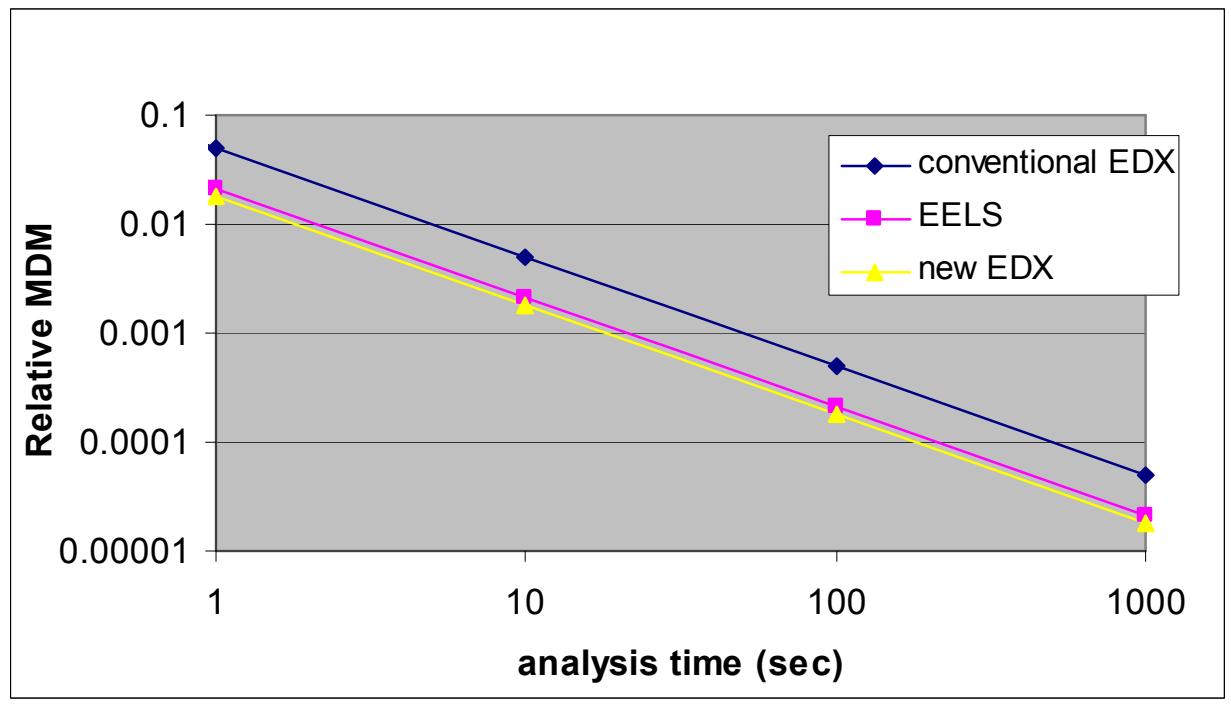

Fig.1 Comparison of relative minimum detectable mass MDM vs. analysis time t by EDX with conventional and new detectors and by EELS (50nm SrTiO3 sample, data source: FEI) 